\title{
SEMI-SIMPLE LOCALLY COMPACT MONOTHETIC SEMI-ALGEBRAS
}

\author{
by M. A. KAASHOEK and T. T. WEST
}

(Received 22nd January 1968)

\section{Introduction}

Bonsall and Tomiuk have shown, in (3), the connection between the local compactness of a monothetic semi-algebra and the spectral properties of a generating element. This theme was developed, in (4), to give a complete characterisation of prime, strict locally compact monothetic semi-algebras in terms of the spectrum of a generator (Theorem $A$ ). Here we extend this result to the case of a semi-simple locally compact monothetic semi-algebra (Theorem $B)$.

In Section 2 we collect the relevant terminology and state the main result. The proofs are given in Section 3. Theorem 1 in Section 3 was suggested to us by $\mathrm{F}$. F. Bonsall. Its use has considerably simplified our original proof.

\section{Terminology and main result}

Let $B$ be a complex Banach algebra with identity $e . R$ will be the set of real numbers, $\boldsymbol{R}^{+}$the set of non-negative real numbers and $\boldsymbol{R}^{++}$the set of strictly positive real numbers. A non-empty subset $A$ of $B$ is called a semialgebra if $x+y, x y$ and $\alpha x$ are in $A$ whenever $x$ and $y$ are in $A$ and $\alpha$ is in $R^{+}$. A semi-algebra $A$ is strict if $A \cap(-A)=(0)$; it is locally compact if $A$ contains non-zero elements and if, in addition,

is a compact subset of $B$.

$$
A \cap\{x:\|x\| \leqq 1\}
$$

The semi-algebra $A$ is said to be monothetic if $A$ has a single generator, that is, if there exists an element $t \in A$ such that $A$ is the closure in $B$ of the set

$$
P(t)=\left\{\alpha_{1} t+\ldots+\alpha_{k} t^{k}: \alpha_{i} \geqq 0(i=1, \ldots, k) ; k=1,2, \ldots\right\} .
$$

In this case we write $A=A(t)$. Obviously, $A(t)$ is commutative.

The commutative semi-algebra $A$ is semi-simple if $a^{2} \neq 0$ for each nonzero $a \in A$, and it is prime if there are no divisors of zero in $A$, in other words, if $a, b \in A$ and $a \neq 0, b \neq 0$ then $a b \neq 0$.

The resolvent set of any element $t \in B$ is denoted by $\rho(t)$. Its complement $\sigma(t)$ in the complex plane $C$ is the spectrum of $t$. The spectral radius of $t$ is denoted by $r(t)$, and the resolvent operator $(z e-t)^{-1}$ by $R(z ; t)$. A point $\lambda \in \sigma(t)$ is called a simple pole of $t$ if it is a pole of the function

of order one.

$$
z \rightarrow R(z ; t)
$$


The following theorem is essentially Theorem 8 in (4).

Theorem A. Let t be a non-zero element of $B . A(t)$ is a prime, strict, locally compact semi-algebra if and only if

and

$$
0<r(t) \in \sigma(t)
$$

$$
\sigma(t) \cap\{\lambda:|\lambda|=r(t)\}
$$

is a finite set of simple poles of $t$.

We now formulate the main result of this paper.

Theorem B. Let $t$ be a non-zero element of $B . A(t)$ is a semi-simple, locally compact semi-algebra if and only if $\sigma(t)$ decomposes uniquely into two disjoint closed subsets $\sigma_{1}$ and $\sigma_{2}$ such that

(i) $\sigma_{1}$ is a finite (possibly empty) set of simple poles of $t$ and

$$
\sigma_{1} \cap R^{++}=\varnothing
$$

(ii) either $\sigma_{2}=\varnothing$ or there exists an $\alpha>0$ in $\sigma_{2}$ such that

and

$$
\sigma_{2}=\sigma(t) \cap\{\lambda:|\lambda| \leqq \alpha\}
$$

$$
\sigma_{2} \cap\{\lambda:|\lambda|=\alpha\}
$$

is a finite set of simple poles of $t$.

Remarks. (a) If we write $t_{2}$ for the " part" of $t$ associated with the spectral set $\sigma_{2}$ then, by Theorem $A, A\left(t_{2}\right)$ is prime, strict and locally compact.

(b) If $\sigma_{1}=\varnothing, A(t)$ is strict. This is a simple consequence of Theorem $A$.

If $A(t)$ is locally compact, strict and semi-simple then by (2), Lemma 8 , $r(t)>0$, and hence a slight modification of Theorem 7 in (4) shows that $A(t)$ is prime. Thus the converse of $(b)$ also follows from Theorem $A$.

\section{Proofs}

Theorem 1. Let $t$ be a non-zero element of $B$. Then $A(t)$ is locally compact and semi-simple if and only if there exists an idempotent $p$ in $A(t)$ such that

(i) $A(t)=A(t p) \oplus A(t-t p)$;

(ii) either $A(t p)=(0)$ or $A(t p)$ is locally compact, semi-simple and

$$
A(t p)=-A(t p)
$$

(iii) either $A(t-t p)=(0)$ or $A(t-t p)$ is locally compact, prime and strict.

Proof of necessity. Suppose that $A(t)$ is locally compact and semi-simple. Put $C=A(t) \cap(-A(t))$; then $C$ is a finite dimensional semi-simple algebra over $\boldsymbol{R}$, and so $C$ contains a unit element $p((\mathbf{1})$ p. 37, Theorem 1). Observing that $t p$ and $t-t p$ are in $A(t)$, and writing $t=t p+(t-t p)$, we have

$$
A(t)=A(t p)+A(t-t p) \text {. }
$$


Suppose that $x \in A(t p) \cap A(t-t p)$. Then there exist $y$ and $z$ in $A(t)$ such that $x=y p=z-z p$. Hence $x=0$. Therefore

and (i) follows.

$$
A(t p) \cap A(t-t p)=(0)
$$

If $x \in C$, then $x=x p$ and hence $x \in A(t p)$. Conversely, take $x \in A(t p)$. Then $x=y p$ for some $y \in A(t)$. Thus $-x=y(-p) \in A(t)$. But then $x \in C$. Hence $C=A(t p)$. This proves (ii).

Suppose that $x$ and $-x$ are in $A(t-t p)$. Then $x \in C=A(t p)$. By (1), $x=0$. Therefore $A(t-t p)$ is strict. It is also locally compact and semi-simple as a subsemi-algebra of $A(t)$. A slight modification of Theorem 7 in (4) shows that it is therefore prime. This proves (iii).

Proof of sufficiency. Let there exist an idempotent $p$ such that conditions (i), (ii) and (iii) hold. Local compactness of $A(t p)$ and $A(t-t p)$ and condition (i) clearly imply that $A(t)$ is locally compact.

Let $a$ in $A(t)$ be such that $a^{2}=0$. Then $(a p)^{2}=a^{2} p=0$ and $a p \in A(t p)$; hence, by (ii), $a p=0$. Similarly $(a-a p)^{2}=a^{2}-a^{2} p=0$ and $a-a p \in A(t-t p)$; hence, by (iii), $a-a p=0$. Thus $a=a p+(a-a p)=0$. Hence $A$ is semi-simple.

Lemma 2. Let $t$ be a non-zero element in $B$. Then $A(t)$ is locally compact, semi-simple and $A(t)=-A(t)$ if and only if $\sigma(t)$ is a finite set of simple poles of $t$ and $\sigma(t) \cap R^{++}$is empty.

Proof of necessity. The hypotheses imply that $A(t)$ is a real finite dimensional semi-simple algebra. So we can apply the Corollary in (1) p. 40 to show that $A(t)$ is algebraically isomorphic to a direct sum of fields, all of which are finite commutative extensions of $\boldsymbol{R}$. But the finite commutative extension fields over $\boldsymbol{R}$ are either copies of $\boldsymbol{R}$ or of $\boldsymbol{C}$. Thus $A(t)$ is algebraically isomorphic to $\boldsymbol{R}^{n} \times \boldsymbol{C}^{m}$ (with coordinate-wise multiplication) for some positive integers $n$ and $m$. We can extend the inverse of this isomorphism to an algebraic homomorphism $\phi$ from $C^{n+m}$ onto the complex Banach algebra $B(t)$ generated by $t$. Let $N$ be the kernel of $\phi$, then $B(t)$ is algebraically isomorphic to the quotient algebra $C^{n+m} / N$. Since $N \neq C^{n+m}, C^{n+m} / N$ is algebraically isomorphic to $C^{r}$ for some positive integer $r$. So there exists an algebraic isomorphism $\psi$ from $B(t)$ onto $C^{r}$. Clearly this implies that the spectrum of $t$ as an element of $B(t)$ is a finite set of simple poles of $t$. Since $t=t p$, where $p$ is the unit of $B(t)$, the spectrum of $t$ as an element of $B$ is a finite set of simple poles of $t$.

Let $\psi(t)=\left(\lambda_{1}, \lambda_{2}, \ldots, \lambda_{k}\right)$. Note that

$$
\sigma(t) \backslash\{0\} \subset\left\{\lambda_{1}, \ldots, \lambda_{k}\right\} .
$$

Suppose that $\sigma(t) \cap R^{++} \neq \varnothing$. Then $\lambda_{i}>0$ for some $i$. This implies that, for any $a$ in $A(t)$, the $i$ th coordinate of $\psi(a)$ is non-negative. In particular, since $-t \in A(t),-\lambda_{i} \geqq 0$ contradicting $\lambda_{i}>0$. This shows that $\sigma(t) \cap R^{++}=\varnothing$.

Proof of sufficiency. Suppose that

$$
\sigma(t)=\left\{\lambda_{1}, \ldots, \lambda_{k}\right\}
$$

is a finite set of simple poles of $t$ such that $(t \sigma) \cap R^{++}=\varnothing$.

$$
\text { E.M.S. }-0
$$


For each $i$, let $p_{i}$ be the spectral idempotent corresponding to $\lambda_{i}$. Then

$$
t=\lambda_{1} p_{1}+\ldots+\lambda_{k} p_{k} \text {. }
$$

Hence each $a$ in $A(t)$ is of the form

$$
a=\alpha_{1} p_{1}+\ldots+\alpha_{k} p_{k}
$$

where $\alpha_{i} \in C(i=1, \ldots, k)$. Therefore $A(t)$ is algebraically isomorphic to a subsemi-algebra of $C^{k}$ with coordinatewise multiplication. Therefore $A(t)$ is locally compact and semi-simple.

Now we can apply Theorem 1 to show the existence of an idempotent $p$ in $A(t)$ such that

(i) $A(t p)=-A(t p)$,

(ii) $A(t-t p)=(0)$ or $A(t-t p)$ is a prime strict locally compact semi-algebra.

Let $\sigma_{1}$ be the spectral set associated with $p$. Standard spectral theory shows that

$$
\sigma(t-t p)= \begin{cases}\left\{\sigma(t) \backslash \sigma_{1}\right\} \cup\{0\} & \text { if } p \neq 0 \\ \sigma(t) & \text { if } p=0 .\end{cases}
$$

Hence $\sigma(t-t p) \cap R^{++}=\varnothing$. If $t-t p \neq 0$, then by (2), Lemma $8, r(t-t p)>0$. Applying Theorem $A$ we get $r(t-t p) \in \sigma(t-t p)$. This contradicts the fact that $\sigma(t-t p) \cap R^{++}=\varnothing$. Hence $t-t p=0$, and thus $A(t)=-A(t)$.

Theorem B. Proof of necessity. Suppose that $A(t)$ is locally compact and semi-simple. Choose $p$ as in Theorem 1 . Since $p$ is a spectral projection, standard spectral theory shows that if $0 \neq \lambda \in \sigma(t p)$ or $0 \neq \lambda \in \sigma(t-t p)$ then $\lambda \in \sigma(t)$, and that, if $\lambda$ is a non-zero simple pole of $t p$ or of $t-t p$, then it is a simple pole of $t$. Also

$$
\{\sigma(t) \cap \sigma(t p)\} \cup\{\sigma(t) \cap \sigma(t-t p)\}=\sigma(t) .
$$

Now suppose that $t-t p \neq 0$. Then, by Theorem $1, A(t-t p)$ is locally compact prime and strict. Hence, by Theorem $A$,

and

$$
0<r(t-t p) \in \sigma(t-t p)
$$

$$
\sigma(t-t p) \cap\{\lambda:|\lambda|=r(t-t p)\}
$$

is a finite set of simple poles of $t-t p$.

Theorem 1 and Lemma 2 show that $\sigma(t p)$ is a finite set of simple poles of $t$ and

Put $\alpha=r(t-t p)$,

$$
\sigma(t p) \cap R^{++}=\varnothing \text {. }
$$

and

$$
\begin{gathered}
\sigma_{1}=\sigma(t) \cap\{\lambda:|\lambda|>\alpha\}, \\
\sigma_{2}=\sigma(t) \cap\{\lambda:|\lambda| \leqq \alpha\} .
\end{gathered}
$$

Then $\alpha>0$ and, by the spectral theory summarized above, $\sigma_{1}$ and $\sigma_{2}$ satisfy the conditions of Theorem $B$. 
If $t-t p=0$, take $\sigma_{1}=\sigma(t)$ and $\sigma_{2}=\varnothing$; again the conditions of Theorem $B$ are satisfied. Clearly, in both cases the decomposition is unique.

Proof of sufficiency. Suppose that $\sigma(t)$ is decomposed into disjoint subsets $\sigma_{1}$ and $\sigma_{2}$ satisfying conditions (i) and (ii) of Theorem B. If $\sigma_{1}=\varnothing$ then $\sigma_{2}=\sigma(t)$. Applying Theorem A we see that $A(t)$ is locally compact and prime, hence also semi-simple.

If $\sigma_{1} \neq \varnothing$, let $p$ be the spectral projection associated with the spectral set $\sigma_{1}$. Then $t p \neq 0$ and $\sigma(t p)$ is a finite set of simple poles of $t p$ such that

$$
\sigma(t p) \cap \boldsymbol{R}^{++}=\varnothing .
$$

So, applying Lemma 2 , we see that $A(t p)$ is locally compact and semi-simple. Now, either $t-t p=0$, or

$$
\sigma(t-t p) \cap\{\lambda:|\lambda|=r(t-t p)\}
$$

is a finite set of poles of $t-t p$ which contains the point $\alpha=r(t-t p)>0$. Hence, either $A(t-t p)=(0)$, or, by Theorem A, $A(t-t p)$ is locally compact, strict and prime. Thus $A(t-t p)$ is also semi-simple. The argument used in the proof of sufficiency of Theorem 1 shows that $A(t)$ is locally compact and semisimple.

\section{REFERENCES}

(1) A. A. Albert, Structure of Algebras (Amer. Math. Soc. Colloquium Publication vol. XXIV, New York, 1939).

(2) F. F. Bonsall, Locally compact semi-algebras, Proc. London Math. Soc. (3) 13 (1963), 51-70.

(3) F. F. Bonsall and B. J. TomiuK, The semi-algebra generated by a compact linear operator, Proc. Edinburgh Math. Soc. (2) 14 (1965), 177-196.

(4) M. A. KAASHOEK and T. T. WEST, Locally compact monothetic semi-algebras, Proc. London Math. Soc. (3) 18 (1968), 428-438.

FreE UNIVERSITY, AMSTERDAM

Trinity College, Dublin 